\title{
Channel Estimation Algorithms for Hybrid Antenna Arrays: Performance and Complexity
}

\author{
Visa Tapio, Mubarak Umar Aminu, Janne Lehtomäki, Markku Juntti \\ Centre for Wireless Communications (CWC) \\ University of Oulu \\ Oulu, Finland \\ E-mail:visa.tapio@oulu.fi,mubarak.aminu@oulu.fi,janne.lehtomaki@oulu.fi, markku.juntti@oulu.fi
}

\begin{abstract}
At the millimeter wave and higher frequency bands the radio channel can often be expressed as a linear combination of a small number of scattering clusters. Hence, the number of angles of arrivals with significant components is limited. Due to severe path losses, the receiver must be equipped with an antenna array capable of forming narrow beams. The channel estimation with narrow beams is challenging. Algorithms developed for sparse estimation problems can be utilized to overcome the problem. In this paper, the performance and computational complexity of channel estimation methods for millimeter and terahertz frequency bands are compared. The methods considered are based on Bayesian learning with the relevance vector machine, orthogonal matching pursuit and the least absolute shrinkage and selection operator optimization. The conventional least squares channel estimator is used as a reference method. The complexity of the least squares estimator is found to be the smallest. The estimation accuracy of the Bayesian learning based estimator is the best but with increased computational complexity.
\end{abstract}

Index Terms-Millimeter wave communications, orthogonal matching pursuit, LASSO, Bayesian learning.

\section{INTRODUCTION}

The abundant spectrum resources at the millimeter and terahertz wave bands are considered to be one of the core capacity increase enablers for the Fifth Generation $(5 G)$ and beyond $5 \mathrm{G}$ wireless networks. The small wave lengths of signals at the frequency bands from tens of gigahertz up to terahertz region allows the realization of antenna arrays with a large number of antenna elements making it feasible to realize high beamforming gains. However, conventional fully digital beamforming architectures cannot be directly applied in millimeter wave communications due to the radio frequency (RF) hardware constraints. Therefore, two-stage digital-analog beamforming architectures (hybrid beamforming) have been proposed [1]. They allow the use of large antenna arrays with a limited number of RF chains.

The estimation of the channel state information (CSI) at at the millimeter and terahertz wave bands is challenging due to narrow beam widths. The least absolute shrinkage and selection operator (LASSO) optimization based channel estimator for millimeter wave hybrid receiver was proposed in [2] and the usage of the orthogonal matching pursuit (OMP) algorithm for the channel estimation was proposed in [3]. Bayesian learning methods has been described in [4] and applied in channel estimation in [5].
In this paper, the performance and complexity of the sparse channel estimation algorithms based on the LASSO, sparse Bayesian learning with Relevance Vector Machine (RVM) and OMP technique are compared. A least squares channel estimator (LS) is used as a reference method.

In theoretical algorithm analysis, the computational complexity is often given using the big $O$ notation. The big $O$ notation is useful when comparing different algorithm classes but it does not allow the feasibility study of the algorithms. At the other extreme, computational complexity can be measured with the number of logic ports and other hardware components needed for the implementation. In order to have a fair comparison in this case, the implementation technology needs to be defined and the same technology must be used for all the algorithms that are being compared. Between these two extremes, the complexity of the algorithms can be measured with the number of operations needed to complete the task the algorithms are designed for. Quite often the complexity is given as the number of elementary mathematical operations (multiplications and additions) and the same metric is used also in this paper. The performance metric for the channel estimation accuracy is the averaged normalized squared error.

The rest of the paper is organized as follows. The system model is described in Section II, algorithms are described in Section III and procedure for the complexity estimation is explained in Section IV. Numerical results are given in Section V and finally, conclusions are presented in Section VI.

\section{SYSTEM MODEL}

The system model is the same as that in [5]. The channel between the antenna arrays at the transmitter and receiver is modeled as

$$
\mathbf{H}=\sum_{l=1}^{L} h_{l} s_{\mathrm{R}}\left(\theta_{l}\right) s_{\mathrm{T}}\left(\phi_{l}\right)
$$

where $s_{\mathrm{R}}\left(\theta_{l}\right)$ and $s_{\mathrm{T}}\left(\phi_{l}\right)$ are the array propagation vectors for transmit directions $\theta_{l}$ and receive directions $\phi_{l}$, respectively and $h_{l}$ are the complex gains of paths $l$.

The channel estimator input is

$$
\mathbf{y}=\left(\mathbf{V}^{T} \mathbf{S}^{*}\left(\Phi_{\mathrm{T}}\right) \otimes \mathbf{W}^{H} \mathbf{S}\left(\Theta_{\mathrm{R}}\right)\right) \mathbf{h}+\mathbf{n}=\mathbf{\Psi h}+\mathbf{n}
$$

where $\mathbf{V}$ and $\mathbf{W}$ are the precoding and combining matrices, respectively. The channel vector $\mathbf{h}$ is a vector form of the 
channel matrix $\mathbf{H}$. Matrices $\mathbf{S}\left(\Phi_{\mathrm{T}}\right)$ and $\mathbf{S}\left(\Theta_{\mathrm{R}}\right)$ consist of steering vector used to scan the transmit and receive directions, respectively.

\section{Channel Estimation Algorithms}

\section{A. Bayesian Learning with Relevance Vector Machine}

The RVM algorithm is originally published in [6] and it can be downloaded from http://www.miketipping.com /sparsebayes.htm\#software. It was applied for channel estimation in [5]. The RVM based channel estimator is given as Algorithm 1. At each iteration one column of the matrix is added to the model, removed from the model or the model is used to update the channel estimate. The selection is done based on the squared absolute value on $q_{i}$ defined as

$$
q_{i}=\Psi_{m}^{\mathrm{H}} \mathrm{C}_{-m}^{-1} \mathbf{y},
$$

where $\Psi$ is the $m^{\text {th }}$ column vector of the matrix $\Psi$ and $\mathrm{C}_{-m}=$ $\sigma^{2} \mathbf{I}\left(\sigma^{2}=\right.$ noise variance).

The details of the RVM based channel estimation can be found from [5].

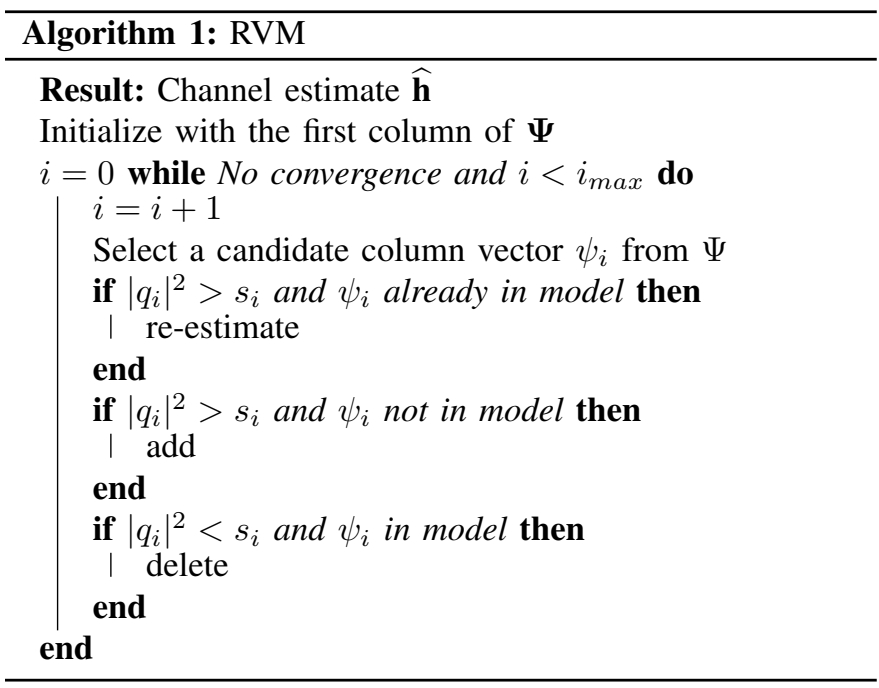

\section{B. LASSO Algorithm}

The LASSO based channel estimation algorithm (Algorithm 2) is the same that was used in [5]. The vector $\mathbf{v}$ in Algorithm 2 is

$$
\mathbf{v}=\left[\begin{array}{c}
\frac{z_{1}^{k}}{\left|z_{1}^{k}\right|^{2}+\epsilon} \\
\vdots \\
\frac{z_{N}^{k}}{\left|z_{N}^{k}\right|^{2}+\epsilon}
\end{array}\right]
$$

$z_{i}^{k}$ is the $i^{\text {th }}$ element of the vector $\mathbf{z}$ at iteration $k$ and $\rho$ is the inverse of the signal-to-noise ratio of the received signal. The parameter $\epsilon$ is used to provide stability [7].

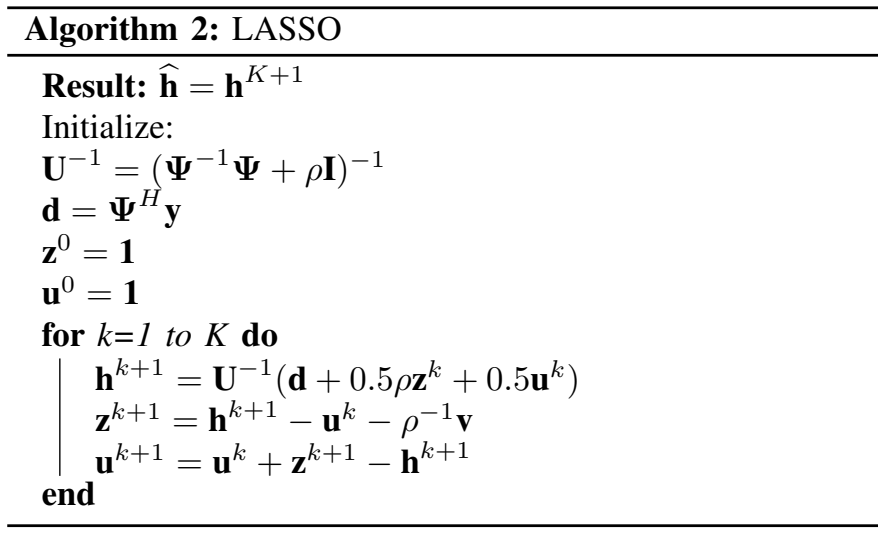

\section{Orthogonal Matching Pursuit Algorithm}

The OMP based channel estimators have been used to estimate channels with sparse channel impulse response [8] as well as to estimate sparse channels in hybrid MIMO systems [3]. Different implementations of the OMP algorithms have been described in [9]. The OMP algorithm used for channel estimation is given as (Algorithm 3). It is used to sequentially identify the rows of the matrix $\Psi$ which correspond to the directions of the received signal.

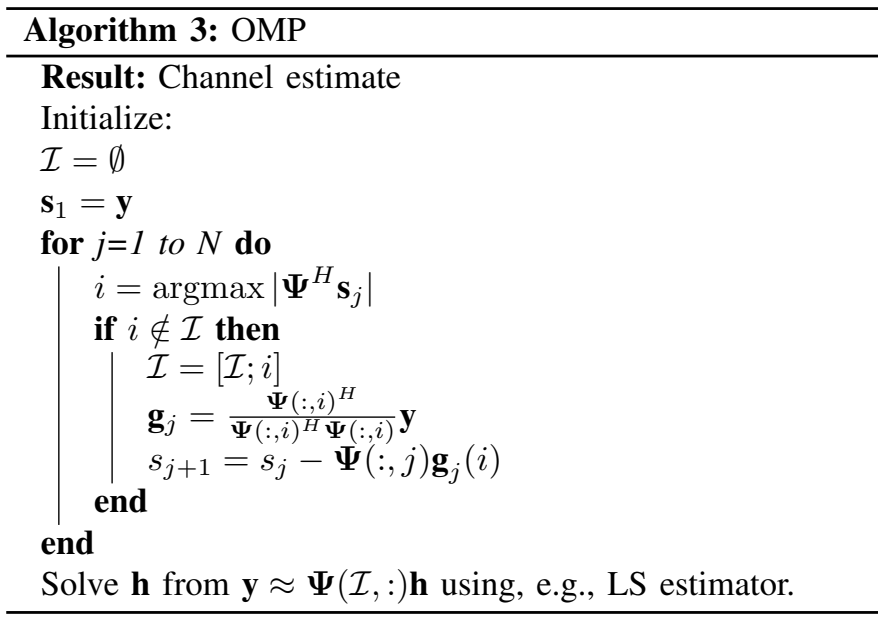

\section{Least Squares Estimation}

The LS estimate is calculated using the matrix $\boldsymbol{\Psi}$ and vector $\mathbf{y}$ as

$$
\mathbf{h}_{\mathrm{LS}}=\left(\boldsymbol{\Psi}^{H} \boldsymbol{\Psi}\right)^{-1} \boldsymbol{\Psi}^{H} \mathbf{y} .
$$

Since the matrix $\Psi$ does not depend on the input signal and its size is constant, it can be calculated off-line and stored in the memory of the device. Hence, the LS estimation consists only of a matrix-vector multiplication.

\section{Estimation ACCURACY AND COMPLEXITY ESTIMATION}

The accuracy of the channel estimator is measured with the averaged normalized estimation error in decibel scale as 


$$
e=10 \log _{10} \frac{1}{N_{\mathrm{mc}}} \sum_{n_{\mathrm{mc}}=0}^{N_{\mathrm{mc}}-1} \frac{\left\|\hat{\mathbf{h}}\left(n_{\mathrm{mc}}\right)-\mathbf{h}\left(n_{\mathrm{mc}}\right)\right\|^{2}}{\left\|\mathbf{h}\left(n_{\mathrm{mc}}\right)\right\|^{2}},
$$

where $\hat{\mathbf{h}}$ and $\mathbf{h}$ are the channel estimate and the true channel, respectively.

The number of iterations and the time spent at $a d d$, reestimate and delete phases of RVM changes randomly. This means that also the number of operations changes from one estimation run to the other. The size of the set $\mathcal{I}$ and hence the size of the LS problem in OMP is also random. In order to assess the computational complexity of these two algorithms, the number of complex multiplications and additions are counted during the simulations. Both algorithms use also other operations than the complex multiplications and additions and some of those operations are expensive from the complexity point of view. However, since the number of those complex operations is much smaller than the number of complex multiplications and additions, the complexity assessment of RVM is based only on the sum of the number of complex multiplications and additions. The OMP algorithm requires also the computation of a LS solution. The LS solution is calculated using the singular value decomposition (SVD). The complexity of SVD as the number of operations is given as [10]

$$
C_{\mathrm{SVD}}=4 m^{2}+8 m n^{2}+9 n^{3},
$$

where $n$ and $m$ are the number of columns and and rows of the matrix in the LS problem, respectively. The total complexity of the OMP algorithm is calculated as the sum of the number of complex multiplications and additions and $C_{\mathrm{SVD}}$.

The complexity of the LASSO algorithm depends on the sizes of the matrix $\Psi$ and vector $y$ and the number of iterations $K$. When these are fixed, the number of operations is constant from one estimation run to the other. The calculation of $\mathbf{U}^{-1}$ is the most computationally intensive part of the LASSO based algorithm since the matrix size to be inverted is large. The matrix inversion is implemented using SVD as in the OMP case. If the SNR of the input signal is known before the channel estimation, the matrix inversion can be calculated before the estimation. In this case the the complexity of the LASSO is reduced considerably. The complexity of the LASSO with and without matrix inversion are counted during simulations.

Since matrix $\Psi$ and hence its pseudo-inverse does not change during the operation, only matrix-vector multiplication is needed in the LS estimation (5).

\section{Numerical RESUlts}

The simulated cases are listed in Table I. The $N_{\text {ant }}$ row shows the number of antenna elements in the antenna array, Clusters row shows the number of reflecting clusters in the channel between the transmitter and receiver, the $O M P$ row gives the number of iterations $N$ in Algorithm 3, the LASSO row gives the number of iterations $K$ used in the Algorithm 2 and $R V M$ row gives the maximum number of iterations in
TABLE I

CASE DEFINITION.

\begin{tabular}{|c|c|c|c|c|}
\hline & Case 1 & Case 2 & Case 3 & Case 4 \\
\hline$N_{\text {ant }}$ & 8 & 8 & 8 & 8 \\
Clusters & 12 & 6 & 2 & 2 \\
OMP & 20 & 20 & 20 & 6 \\
LASSO & 5 & 5 & 5 & 5 \\
RVM & 17 & 17 & 17 & 6 \\
\hline
\end{tabular}

Algorithm 1. The size of the matrix $\Psi$ and hence the computational complexity of the estimation depends on the number of antennas. In a 8 antenna element case the size of the matrix $\Psi$ is $64 \times 256$ and the length of the estimated channel vector $\mathbf{h}$ is 64 .

In the Case 1, the channel consists of relatively high number of reflection clusters. The parameters in Table I are chosen so that the accuracy of the estimators is maximized, i.e., increasing the complexity from these values does not improve the estimation accuracy. These values have been found experimentally by simulations. The estimation accuracy of the algorithms in Case 1 are shown in Fig 1. In Cases 2 and 3 , the number of reflecting clusters in the channel model is reduced to 6 and 2, respectively. The parameters controlling the complexity of the algorithms are the same as in Case 1 . Estimation accuracy in Cases 2 and 3 are shown in Figs. 2 and 3. In Case 4, the number of clusters is kept the same as in Case 3 but the parameters controlling the complexity of OMP and RVM are minimized, i.e., if the parameter values were smaller the estimation accuracy would be impaired or the estimation would fail completely. The estimation accuracy of the algorithms in Case 4 is shown in Fig. 4. The number of iterations for the LASSO algorithm is 5 in all the cases. Increasing the value from this did not improve the estimation accuracy significantly in any of the cases.

When the estimator parameters (OMP, LASSO, RVM) in Table I are kept the same (Cases 1-3), the estimation accuracy of the algorithms does not change regardless the number of reflecting clusters in the channel. Only exception is the OMP algorithm whose performance at SNR values above $5 \mathrm{~dB}$ in Case 1 is worse than in Cases 2 and 3. When the complexity parameter values for the OMP and RVM algorithms are selected based on the number of clusters in Case 4, their performance is improving. Especially, the performance of the OMP algorithm is approaching the performance of the RVM, which has the best performance in all the cases. The average complexities of the algorithms in Cases 1-4 are given in Table II. The complexity of the LS estimator is fixed and depends only on the size of matrix $\Psi$ and the length of vector y. The complexity of the LASSO algorithm depends also on the number of iterations $K$. But since $K$ is the same in all the case, the complexity of LASSO is also constant. As can be seen in Table II, the inversion of the matrix $U$ is increases the complexity of LASSO significantly. Since the number of operations in the OMP and LASSO algorithms is random, the mean and standard deviation (std) values in all the cases at different SNR values are given in Table III. The std values 0 
TABLE II

COMPUTATIONAL COMPLEXITY.

\begin{tabular}{|c|c|c|c|c|}
\hline & Case 1 & Case 2 & Case 3 & Case 4 \\
\hline OMP & $6.7 \cdot 10^{5}$ & $6.9 \cdot 10^{5}$ & $7.0 \cdot 10^{5}$ & $1.6 \cdot 10^{5}$ \\
LASSO & $3.5 \cdot 10^{8}$ & $3.5 \cdot 10^{8}$ & $3.5 \cdot 10^{8}$ & $3.5 \cdot 10^{8}$ \\
LASSO no SVD & $4.1 \cdot 10^{5}$ & $4.1 \cdot 10^{5}$ & $4.1 \cdot 10^{5}$ & $4.1 \cdot 10^{5}$ \\
RVM & $4.8 \cdot 10^{5}$ & $4.8 \cdot 10^{5}$ & $4.7 \cdot 10^{5}$ & $2.3 \cdot 10^{5}$ \\
LS & $3.5 \cdot 10^{4}$ & $3.5 \cdot 10^{4}$ & $3.5 \cdot 10^{4}$ & $3.5 \cdot 10^{4}$ \\
\hline
\end{tabular}

TABLE III

COMPUTATIONAL COMPLEXITY STATISTICS FOR OMP AND RVM.

\begin{tabular}{|c|c|c|c|c|c|}
\hline OMP & SNR & Case 1 & Case 2 & Case 3 & Case 4 \\
\hline mean & $-5 \mathrm{~dB}$ & $7.0 \cdot 10^{5}$ & $6.9 \cdot 10^{5}$ & $7.0 \cdot 10^{5}$ & $1.6 \cdot 10^{5}$ \\
& $0 \mathrm{~dB}$ & $6.8 \cdot 10^{5}$ & $6.9 \cdot 10^{5}$ & $7.0 \cdot 10^{5}$ & $1.6 \cdot 10^{5}$ \\
& $5 \mathrm{~dB}$ & $6.5 \cdot 10^{5}$ & $6.9 \cdot 10^{5}$ & $7.0 \cdot 10^{5}$ & $1.6 \cdot 10^{5}$ \\
& $10 \mathrm{~dB}$ & $6.6 \cdot 10^{5}$ & $6.9 \cdot 10^{5}$ & $7.0 \cdot 10^{5}$ & $1.6 \cdot 10^{5}$ \\
& $15 \mathrm{~dB}$ & $6.7 \cdot 10^{5}$ & $7.0 \cdot 10^{5}$ & $7.0 \cdot 10^{5}$ & $1.6 \cdot 10^{5}$ \\
& $20 \mathrm{~dB}$ & $6.7 \cdot 10^{5}$ & $6.9 \cdot 10^{5}$ & $7.0 \cdot 10^{5}$ & $1.6 \cdot 10^{5}$ \\
\hline std & $-5 \mathrm{~dB}$ & $0.4 \cdot 10^{5}$ & $7.3 \cdot 10^{4}$ & 0 & 0 \\
& $0 \mathrm{~dB}$ & $0.8 \cdot 10^{5}$ & $5.3 \cdot 10^{4}$ & 0 & 0 \\
& $5 \mathrm{~dB}$ & $1.1 \cdot 10^{5}$ & $6.5 \cdot 10^{4}$ & $3.5 \cdot 10^{4}$ & 0 \\
& $10 \mathrm{~dB}$ & $1.1 \cdot 10^{5}$ & $6.0 \cdot 10^{4}$ & $0.5 \cdot 10^{4}$ & 0 \\
& $15 \mathrm{~dB}$ & $0.9 \cdot 10^{5}$ & $5.4 \cdot 10^{4}$ & 0 & 0 \\
& $20 \mathrm{~dB}$ & $0.9 \cdot 10^{5}$ & $8.0 \cdot 10^{4}$ & 0 & 0 \\
\hline RVM & $\mathrm{SNR}$ & Case 1 & Case 2 & Case 3 & Case 4 \\
\hline mean & $-5 \mathrm{~dB}$ & $4.8 \cdot 10^{5}$ & $4.8 \cdot 10^{5}$ & $4.8 \cdot 10^{5}$ & $2.3 \cdot 10^{5}$ \\
& $0 \mathrm{~dB}$ & $4.8 \cdot 10^{5}$ & $4.8 \cdot 10^{5}$ & $4.7 \cdot 10^{5}$ & $2.3 \cdot 10^{5}$ \\
& $5 \mathrm{~dB}$ & $4.8 \cdot 10^{5}$ & $4.8 \cdot 10^{5}$ & $4.7 \cdot 10^{5}$ & $2.3 \cdot 10^{5}$ \\
& $10 \mathrm{~dB}$ & $4.8 \cdot 10^{5}$ & $4.8 \cdot 10^{5}$ & $4.7 \cdot 10^{5}$ & $2.3 \cdot 10^{5}$ \\
& $15 \mathrm{~dB}$ & $4.8 \cdot 10^{5}$ & $4.8 \cdot 10^{5}$ & $4.7 \cdot 10^{5}$ & $2.3 \cdot 10^{5}$ \\
& $20 \mathrm{~dB}$ & $4.8 \cdot 10^{5}$ & $4.8 \cdot 10^{5}$ & $4.7 \cdot 10^{5}$ & $2.3 \cdot 10^{5}$ \\
\hline std & $-5 \mathrm{~dB}$ & $1.3 \cdot 10^{4}$ & $1.2 \cdot 10^{4}$ & $1.2 \cdot 10^{4}$ & $1.0 \cdot 10^{4}$ \\
& $0 \mathrm{~dB}$ & $1.2 \cdot 10^{4}$ & $1.4 \cdot 10^{4}$ & $1.3 \cdot 10^{4}$ & $1.0 \cdot 10^{4}$ \\
& $5 \mathrm{~dB}$ & $1.5 \cdot 10^{4}$ & $1.3 \cdot 10^{4}$ & $1.3 \cdot 10^{4}$ & $1.0 \cdot 10^{4}$ \\
& $10 \mathrm{~dB}$ & $1.5 \cdot 10^{4}$ & $1.4 \cdot 10^{4}$ & $1.3 \cdot 10^{4}$ & $1.0 \cdot 10^{4}$ \\
& $15 \mathrm{~dB}$ & $1.4 \cdot 10^{4}$ & $1.3 \cdot 10^{4}$ & $1.4 \cdot 10^{4}$ & $1.0 \cdot 10^{4}$ \\
& $20 \mathrm{~dB}$ & $1.9 \cdot 10^{4}$ & $1.2 \cdot 10^{4}$ & $1.5 \cdot 10^{4}$ & $1.0 \cdot 10^{4}$ \\
\hline
\end{tabular}

for the OMP algorithm means that with the chosen parameter values the size of the set $\mathcal{I}$ the algorithm has produced is the same for all the channel realizations.

\section{CONCLUSION}

Four different methods for the channel estimation with hybrid antenna arrays were compared. The RVM based estimator gives the best estimation accuracy in all the tested cases. When the number of iterations in the OMP algorithm is selected based on the number of reflecting clusters in the channel, its performance approaches the performance of the RVM, especially when the number of clusters is small. When the number of iterations in OMP increases its performance approaches the performance of the LS estimator. If the number of iterations in OMP were the same as the number of rows of matrix $\Psi$, it would be identical with the LS algorithm. The performance of the LASSO based algorithm is always the worst.

Since matrix $\Psi$ does not depend on the received signal, the calculation of the LS estimation consists of only matrix-vector multiplication. Despite the large matrix size, the computational complexity of the LS estimator is the smallest. The RVM based channel estimator has lower complexity than the OMP when

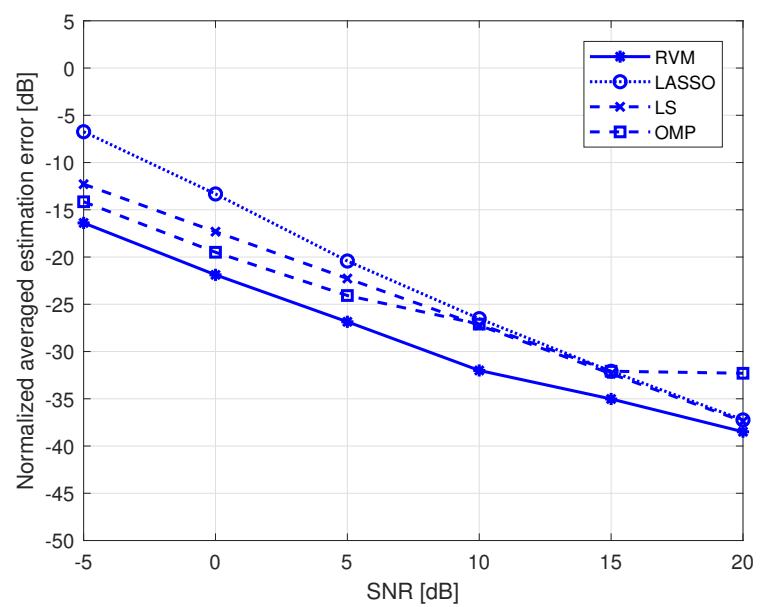

Fig. 1. Channel estimation accuracy in Case 1.

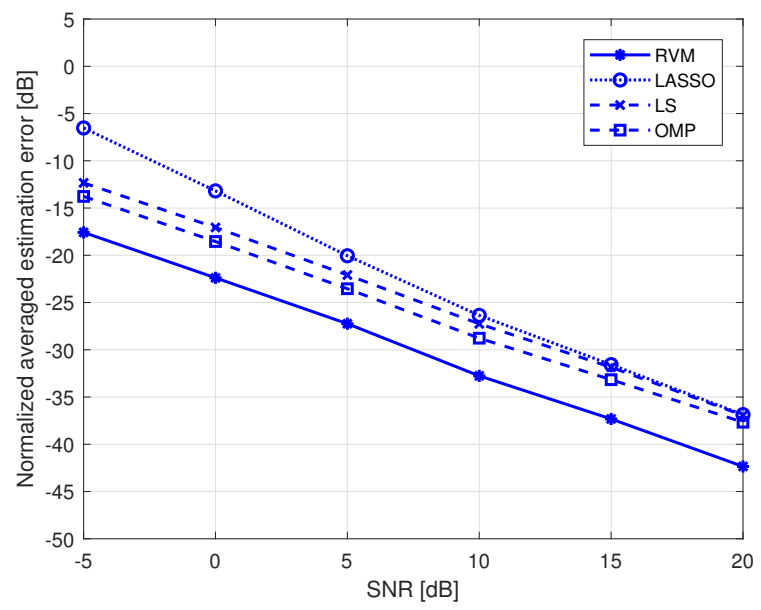

Fig. 2. Channel estimation accuracy in Case 2.

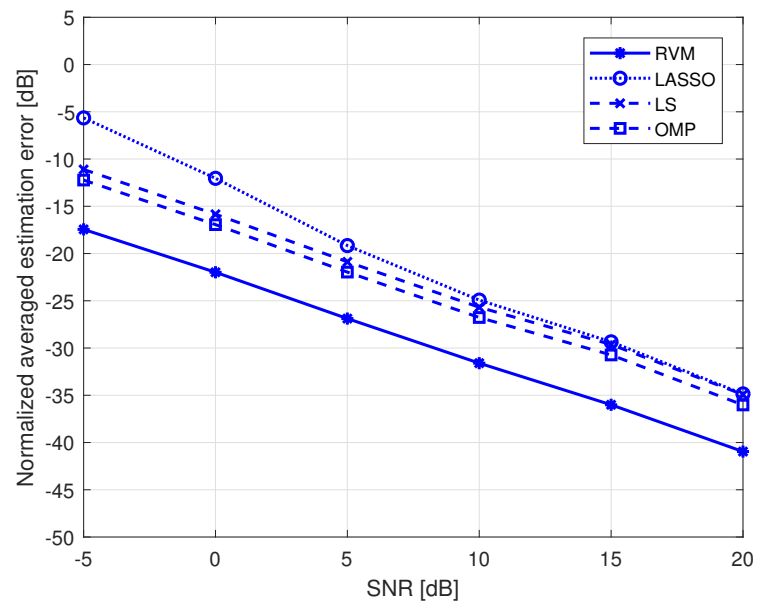

Fig. 3. Channel estimation accuracy in Case 3. 


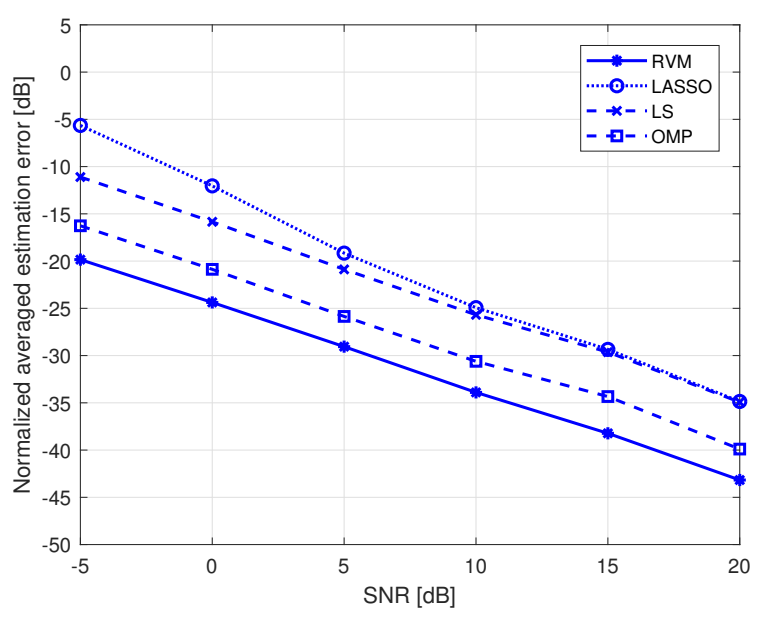

Fig. 4. Channel estimation accuracy in Case 4.

only complex multiplications and additions and the calculation of the SVD in OMP are taken into account. The RVM requires also some more complex functions, such as logarithms, to be calculated. Their portion is, however, small. Therefore, the overall complexity of the RVM is not increased by them significantly. One issue with the RVM is the computation time which can vary from one estimation to the other depending on how much time is spent in add, delete or re-estimate branches of the algorithm.

Based on the results from Cases 1-4, the practical choice is between the LS estimator or RVM based estimator. The LS estimate is the best for low power implementations if its accuracy is good enough for the application, RVM is the best choice when the computational complexity is not as important.

\section{ACKNOWLEDGMENT}

This work has received funding from Horizon 2020, European Union's Framework Programme for Research and Innovation, under grant agreement No. 761794 (TERRANOVA Project) and from Academy of Finland 6Genesis Flagship (grant 318927).

\section{REFERENCES}

[1] A. Alkhateeb, J. Mo, N. Gonzalez-Prelcic, and R. W. Heath, "Mimo precoding and combining solutions for millimeter-wave systems," IEEE Communications Magazine, vol. 52, no. 12, pp. 122-131, December 2014.

[2] A. Alkhateeb, O. El Ayach, G. Leus, and R. W. Heath, "Channel estimation and hybrid precoding for millimeter wave cellular systems," IEEE Journal of Selected Topics in Signal Processing, vol. 8, no. 5, pp. 831-846, Oct 2014.

[3] J. Lee, G. Gil, and Y. H. Lee, "Channel estimation via orthogonal matching pursuit for hybrid mimo systems in millimeter wave communications," IEEE Transactions on Communications, vol. 64, no. 6, pp. 2370-2386, June 2016.

[4] M. E. Tipping, "Sparse bayesian learning and the relevance vector machine," Journal of Machine Learning Research, vol. 1, pp. 211-244, 2001.

[5] M. Umar Aminu, M. Codreanu, and M. Juntti, "Bayesian learning based millimeter-wave sparse channel estimation with hybrid antenna array," in 2018 IEEE 19th International Workshop on Signal Processing Advances in Wireless Communications (SPAWC), June 2018.
[6] M. E. Tipping and A. Faul, "Fast marginal likelihood maximisation for sparse bayesian models," in Proceedings of the Ninth International Workshop on Artificial Intelligence and Statistics, January 2001.

[7] Emmanuel J. Candès, Michael B. Wakin, and Stephen P. Boyd, "Enhancing sparsity by reweighted 1 minimization," Journal of Fourier Analysis and Applications, vol. 14, no. 5, pp. 877-905, Dec 2008.

[8] X. Jiang, W. Zeng, and E. Cheng, "A fast algorithm for sparse channel estimation via orthogonal matching pursuit," in 2011 IEEE 73rd Vehicular Technology Conference (VTC Spring), May 2011, pp. $1-5$.

[9] B. L. Sturm and M. G. Christensen, "Comparison of orthogonal matching pursuit implementations," in 2012 Proceedings of the 20th European Signal Processing Conference (EUSIPCO), Aug 2012, pp. 220-224.

[10] G. H. Golub and C. F. van Loan, Matrix Computations, The Johns Hopkins University Press, third edition, 1996. 